\title{
INVESTIGATION OF ERROR CONCEALMENT USING DIFFERENT TRANSFORM CODINGS AND MULTIPLE DESCRIPTION CODINGS
}

\author{
Ali Farzamnia* - Sharifah K. Syed-Yusof* \\ — Norsheila Fisal ${ }^{*}$ - Syed A. R. Abu-Bakar ${ }^{* *}$
}

\begin{abstract}
There has been increasing usage of Multiple Description Coding (MDC) for error concealment in non-ideal channels. A lot of ideas have been masterminded for MDC method up to now. This paper described the attempts to conceal the error and reconstruct the lost descriptions caused by combining MDC and lapped orthogonal transform (LOT). In this work LOT and other transforms codings (DCT and wavelet) are used to decorrelate the image pixels in the transform domain. LOT has better performance at low bit rates in comparison to DCT and wavelet transform. The results show that MSE for the proposed methods in comparison to DCT and wavelet have decreased significantly. The PSNR values of reconstructed images are high. The subjective evaluation of image is very good and clear. Furthermore, the standard deviations of reconstructed images are very small especially in low capacity channels.

K e y w o r ds: multiple description coding, wavelet transform, lapped orthogonal transform, discrete cosine transform
\end{abstract}

\section{INTRODUCTION}

Multiple Description Coding (MDC) is a source coding $j$ technique that is robust against inevitable transmission errors. MDC encodes a media source into two or more sub-bit streams (descriptions) that are of equal importance. These descriptions can be decoded independently to produce a signal of basic quality. When more descriptions are received, the decoder can gradually increase the quality. The insertion of a certain amount of redundancy in the descriptions among the stream is the cost of this operation. However, redundancy in different descriptions is utilized to estimate the loss description when packet loss happens. There are several methods for applying multiple description coding, but it was founded basically two groups are more common in literature. One is Multiple Description Scalar Quantizer (MDSQ) [1-3] and the other is Multiple Description Transform Coding (MDTC) $[4,5]$.

DCT has been a very popular transform for many years. The fact that DCT is a near optimal transform is the main reason for its popularity. The optimal transform up to now is Karhunen Loeve Transform (KLT). Though it is optimal, it is not widely adopted since it is very complicated and slow. There have been some new algorithms proposed to improve the KLT to make it less complex $[6,7]$, still the complexity in KLT is considered high. DCT, the closest to KLT, is much faster to compute. DCT is also very closely related to the Discrete Fourier Transform (DFT). It is actually possible to compute DCT using DFT [8].

The main difference between DCT and DFT is that DCT only has real values which make it comparatively easier to compute. In recent years, the wavelet transform has been often employed in transformed image coders. The wavelet transform reduces the entropy of the image just like other linear transforms. It means that the wavelet coefficient map has lower entropy than the image. Compared to the DCT, the wavelet transform allows better localization in both frequency and space [9]. Blocking artifacts are omitted in a wavelet based coder.

A new class of orthogonal transforms is called lapped transforms, which are developed by [10] that have been applied in a vast majority of areas such as filtering, image and speech coding, spectral estimation and many others where a traditional block transform is employed. These lapped transforms are developed to overcome the block artifacts that have been so common in traditional block transforms like the DCT [9-11]. Besides reducing the block artifacts, the lapped transforms have fast computational algorithms. The main point is that the LOT has higher coding gains than the DCT.

In this paper joint MDC with LOT is proposed to eliminate errors, which occur in channels during transmission. The goal of using LOT is to change the correlated pixels of the image to independent symbols in mapped domain. The advantage of using LOT is that it has high performance in low bit rates, which is useful in low capacity channels. So in this case, lots of redundant data can be eliminated. Then by using MDC, independent and equal information of descriptions are transmitted to the receiver. If some of the received descriptions are corrupted, other safe received descriptions are able to conceal errors. Thus achieving data are robustness along an aggressive channel.

In Section $2 \mathrm{MDC}$ is elaborated and in Section 3 lapped orthogonal transform is explained. In Section 4

Faculty of Electrical Engineering, Universiti Teknologi Malaysia, Skudai, Johor Bahru, 81310 Johor, Malaysia, ${ }^{*}$ MIMOS Center of Excellence in Telecommunication Technology, ${ }^{* *}$ Dept. of Microelectronics \& Computer Engineering; alifarzamnia@gmail.com, $\{$ kamilah,Sheila,syed\}@fke.utm.my 
investigation results are presented and finally Section 5 describes the paper conclusion.

\section{MULTIPLE DESCRIPTION CODING (MDC)}

A transform based image coder consists of a transform, which maps the spatial domain image representation to the transform domain for better decorrelation; a quantizer, which quantizes the transform coefficients to achieve lossy compression; and an entropy coder, which removes the remaining statistical redundancy between quantized coefficients in a lossless manner. MDC methods use various possibilities for adding redundancy to data. Some of them employ subsampling in spatial or transform domain, while others apply transforms to create interleaved patterns or special scanning and quantization of transform coefficients [11]. The block diagram of MDC is represented in Fig. 1. At first, image is divided into two or more descriptions, which are then being transmitted along the channel. At the receiver, a high quality of image can be reconstructed by MDC even though several descriptions are lost during the transmission [12]. If a good description is designed at a rate $R_{1}$ is sent over channel 1 and another good description is sent over channel 2 at a rate $R_{2}$. The signal reconstructed from either individual description has the least distortion for that rate. However, there is no reason to believe that this is a good way to allot bits for the central description at rate $R_{1}+R_{2}$ similarly; a good compressed representation at rate $R_{1}+R_{2}$ is hard to be split into two good single descriptions. The central theoretical problem of MD model is to determine, the set of achievable values for the quintuple $\left(R_{1} ; R_{2} ; D_{0} ; D_{1} ; D_{2}\right)$ for a given source and distortion measure. The work of El Gamal and Cover [12] gives one derivation of the theoretical boundary of $\left(R_{1} ; R_{2} ; D_{0} ; D_{1} ; D_{2}\right)$.

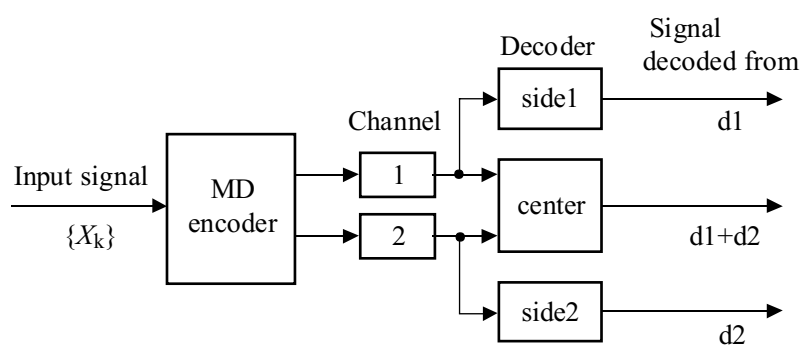

Fig. 1. Block diagram of multiple description coding

Let a sequence of independent identically distributed random variables $x=\left(x_{1}, x_{2}, \ldots, x_{n}\right)$ have two side descriptions denoted as $\tilde{x}_{1}$ and $\tilde{x}_{2}$. The central description is denoted as $\tilde{x}_{0}$ It has been established in [12] that the central distortion and side distortions $D_{0} D_{1}$ and $D_{2}$ are achievable if there exists a probability mass distribution $p\left(\tilde{x}_{1}, \tilde{x}_{2}, \tilde{x}_{0} \mid x\right) p(x)$ with $E\left\{d\left(x, \tilde{x}_{m}\right)\right\}<D_{m}, m=0,1,2$ such that

$$
\begin{gathered}
R_{1}>I\left(x ; \tilde{x}_{1}\right), \quad R_{2}>I\left(x ; \tilde{x}_{2}\right), \\
R_{1}+R_{2}>I\left(x ; \tilde{x}_{1}, \tilde{x}_{2}, \tilde{x}_{0}\right)+I\left(\tilde{x}_{1} ; \tilde{x}_{2}\right)
\end{gathered}
$$

where $I$ denotes Shannon mutual information [13]. Some achievable quintuples $\left(R_{1} ; R_{2} ; D_{0} ; D_{1} ; D_{2}\right)$ may not be within the boundary specified by equation (1). The boundary defined by El Gamal and Cover is called an inner bound. The MD achievable region is completely known only for memoryless Gaussian sources and it is shown by Ozarow [14] that the achievable region for a memory less Gaussian source with unit variance is the largest set that can be obtained with boundary derived by El Gamal and and Cover, which is

$$
\begin{gathered}
D_{1} \geq 2^{-2 R_{1}}, \quad D_{2} \geq 2^{-2 R_{2}}, \\
D_{0} \geq 2^{-\left(R_{1}+R_{2}\right)} \gamma\left(D_{1}, D_{2}, R_{1}, R_{2}\right)
\end{gathered}
$$

$$
\gamma=\frac{1}{1-\left(\sqrt{\left(1-D_{1}\right)\left(1-D_{2}\right)}-\sqrt{D_{1} D_{2}-2^{-2\left(R_{1}+R_{2}\right)}}\right)^{2}}
$$

for $D_{1}+D_{2}<1+2^{-2\left(R_{1}+R_{2}\right)}$, and otherwise $\gamma=1$.

The above equations are interpreted in three situations [15]:

- The side descriptions are very good individually: $D_{1}=$ $2^{-2 R_{1}}$ and $D_{2}=2^{-2 R_{2}}$. Then,

$D_{0} \geq D_{1} D_{2} \frac{1}{1-\left(1-D_{1}\right)\left(1-D_{2}\right)}=\frac{D_{1} D_{2}}{D_{1}+D_{2}-D_{1} D_{2}}$

which leads to $D_{0} \geq \min \left(D_{1}, D_{2}\right) / 2$.

- The central description is as good as possible

$$
D_{0}=2^{-2\left(R_{1}+R_{2}\right)} \text { Then } D_{1}+D_{2}=2^{-2\left(R_{1}+R_{2}\right)} .
$$

- Intermediate between the above two extreme cases: The situation is analyzed for the balanced case. Under the assumptions $R_{1}=R_{2} \gg 1$ therefore $D_{1}=D_{2} \ll$ $1, \frac{1}{\gamma}=1-\left(\left(1-D_{1}\right)-\sqrt{D_{1}^{2}-2^{-4 R_{1}}} \approx 4 D_{1}, D_{0} \geq\right.$ $2^{-4 R_{1}}\left(4 D_{1}\right)^{-1}$. Then,

$$
D_{0} D_{1} \geq \frac{1}{4} 2^{-4 R_{1}}
$$

An easy way for adding redundancy to the compressed image bit stream is to conduct at the quantization stage. Multiple description scalar quantization (MDSQ) has been developed by Vaishampayan [1]. MDSQ works as follows. Two side (coarse) quantizers function in parallel at the quantization stage. The quantized source can be reconstructed from the output of either quantizer with lower quality or from both quantizers in higher quality. When the outputs of two quantizers are joined together, they create higher quality reconstruction due to smaller quantization cells.

In practical, the encoder first uses a scalar quantizer. At this stage, input variables are mapped to a quantization index $I$ [1]. Then, an index assignment is applied and mapped each index $I$ to a codeword index pair $(i, j)$ in 
a codebook. Figure 2a presents the index assignment matrix with spread 2. The cells of the quantizer matrix are numbered from -7 to 7 . The row and column indices of the index assignment matrix form the index pair $(i, j)$. Index $i$ is included in Description 1 and index $j$ is included in Description 2. The central decoder reconstructs the best quality of the original input image. The side decoders estimate somehow low quality of the image. In Fig.2a, only 15 out of 64 cells in the index assignment matrix are occupied. Unoccupied cells constitute coding redundancy. Figure $2 \mathrm{~b}$ shows the index assignments with three diagonals (spread 3) being filled, hence lower redundancy.

\begin{tabular}{|c|c|c|c|c|c|c|c|c|}
\hline & -3 & -2 & -1 & 0 & 1 & 2 & 3 & 4 \\
\hline-4 & -7 & & & & & & & \\
-3 & -6 & -5 & & & & & & \\
\hline-2 & & -4 & -3 & & & & & \\
-1 & & & -2 & -1 & & & & \\
0 & & & & 0 & 1 & & & \\
1 & & & & & 2 & 3 & & \\
\hline 2 & & & & & & 4 & 5 & \\
\hline 3 & & & & & & & 6 & 7 \\
\hline
\end{tabular}

\begin{tabular}{|c|c|c|c|c|c|c|c|c|}
\hline & -3 & -2 & -1 & 0 & 1 & 2 & 3 & 4 \\
\hline-4 & -10 & -9 & & & & & & \\
\hline-3 & -8 & -7 & -5 & & & & & \\
\hline-2 & & -6 & -4 & -3 & & & & \\
\hline-1 & & & -2 & -1 & 1 & & & \\
\hline 0 & & & & 0 & 2 & 3 & & \\
\hline 1 & & & & & 4 & 5 & 7 & \\
\hline 2 & & & & & & 6 & 8 & 9 \\
\hline 3 & & & & & & & 10 & 11 \\
\hline
\end{tabular}

Fig. 2. (a) Index assignment spread 2, (b) Index assignment spread 3

\section{LAPPED ORTHOGONAL TRANSFORM (LOT)}

The key to the development of LOT is the recognition that the blocking effects are caused by the discontinuities on the basis functions of the transforms $[16,17]$. The LOT basis functions of the neighboring blocks overlap as is shown in Fig. 3.

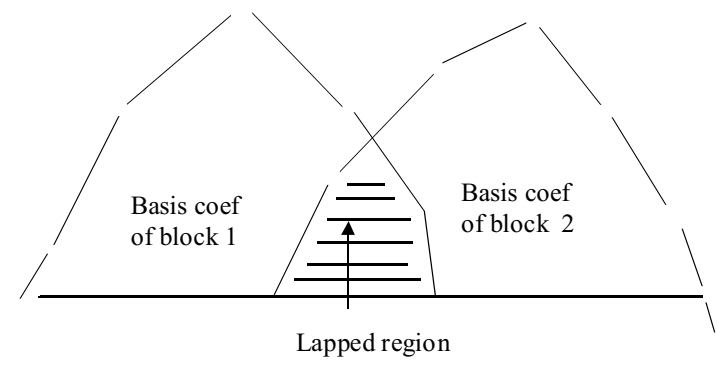

Fig. 3. Lapped basis functions
The basis functions which are the rows of the LOT matrix represent a set of basis information, and an image is nothing but a linear combination of all these information. So when these basis functions of the neighboring block overlap, image distortion occurs. It is decided that the basis functions of the transform can be made longer than the transform length so that the basis functions could have a smooth transition to and from zero at their ends. As a result, the basis functions of the LOT can be extended from the current block to the neighboring blocks on both sides. Because the basis functions are now overlapped between two neighboring blocks, these transforms are named lapped transforms. Also the overlapping portions of the basis functions of the neighboring blocks should be orthogonal if the same basis functions are to be used for both analysis (direct transform) and synthesis (inverse transform). In standard block transforms, the input signal is divided into blocks of $\mathrm{N}$ samples. These $\mathrm{N}$ samples are transformed by an orthogonal matrix of order $N$, where $N$ is the block size, or the number of transform coefficients. With the lapped transform, the $L$ sample input block is mapped into $N$ transform coefficients, where $L$ is the length of the LOT basis functions, with $L>N$. To maintain the total sampling rate, $N$ new transform coefficients for every new $N$ input samples are computed. After the computation of the LOT of a signal block, the $N$ samples of the new block are shifted into the $L$-sample input buffer so that there will be an overlap of $L-N$ samples in the computation of consecutive LOT blocks. It is assumed that $L=2 N[16,17]$, where the basis functions of the LOT are the columns of a $L \times N$ matrix $P$. Here $P$ and $P^{\top}$ are the direct and inverse transform operators. Since the direct transform operators are overlapped, the outputs of each inverse transform operator is also overlapped for perfect signal reconstruction. The reconstructed samples from different blocks are simply added in the regions of overlap. The difference between the regular transform and lapped transform is that when an inverse LOT is applied to the set of transformed coefficients, $L$ samples that are not equal to the original $L$ samples that were used to compute the direct LOT were obtained. So only after the overlapping of consecutive inverse LOT blocks, the original signal would be recovered. An optimal LOT should minimize the bit rate for any reconstruction error level. This is equivalent to maximizing the energy compaction measure or maximum transform coding gain $G_{T C}$ [18] given by

$$
G_{T C}=\frac{\frac{1}{N} \sum_{i=1}^{N} \sigma_{i}^{2}}{\left(\prod_{i=1}^{N} \sigma_{i}^{2}\right)^{\frac{1}{N}}}
$$

where $\sigma_{i}^{2}$ are the diagonal entries of the matrix $R_{0}$ that is defined in equation (8).

An optimal LOT is obtained by an iterative optimization technique that searches for a maximum of $G_{T C}[19]$. It has the disadvantage of being highly sensitive to numerical errors. So in [20], a direct approach is derived for an 
optimal LOT when the basis functions of the neighboring blocks overlapped by $N$ samples, $i e$ when $L=2 N$. Since the lower order basis functions of consecutive blocks are super imposed, a constant level is obtained and this flat field can be transformed into one LOT coefficient. This property is important for energy compaction and hence compression. Let $R_{x x}$ be the autocorrelation matrix for the first order Markov process given by

$$
R_{x x}=\left[\begin{array}{ccccc}
1 & \rho & \rho^{2} & \ldots & \rho^{L} \\
\rho & 1 & \rho & \ldots & \rho^{L-1} \\
\vdots & \ddots & \ddots & \ddots & \vdots \\
\rho^{L-1} & \ldots & \rho & 1 & \rho \\
\rho^{L} & \ldots & \rho^{2} & \rho & 1
\end{array}\right]
$$

where $\rho$ is the correlation coefficient between adjacent samples. Any matrix $P_{0}$ that diagonals the autocorrelation matrix $R_{x x}$ would be a feasible LOT. Defining $R_{0}$ as

$$
\begin{aligned}
R_{0} & =P_{0}^{\top} R_{x x} P_{0}=Z^{\top} P^{\top} R_{x x} P Z, \\
R^{\prime} & =P^{\top} R_{x x} P
\end{aligned}
$$

where $Z$ is any orthogonal matrix of size $N \times N$ so $Z^{\top} Z=I$. Therefore, $Z$ is derived such that it will diagonalize the matrix $P^{\top} R_{x x} P$ to give an exact solution for $Z$. Given a DCT, a feasible LOT can be defined as

$$
P=\frac{1}{2}\left[\begin{array}{cc}
D_{e}-D_{o} & D_{e}-D_{o} \\
j\left(D_{e}-D_{o}\right) & -j\left(D_{e}-D_{o}\right)
\end{array}\right]
$$

where $D_{e}$ and $D_{o}$ are the $N \times \frac{N}{2}$ matrices containing the even and odd DCT functions respectively and $j$ is the $N \times N$ counter identity matrix [21]. All the properties of a feasible LOT that are discussed before are satisfied by the $P$ matrix. Using $P, Z$ can be obtained as the KLT matrix of $R_{0}$ for a given correlation coefficient ( $\rho=$ $0.95)$. This optimization approach leads to an optimal LOT that is related to the choice of the initial matrix $P$. Each column of $P$ has $L$ elements, with $L>N$, spanning in $N$ dimensional subspace. For any $Z$, the matrix $P Z$ will also be in the same subspace and hence the LOT is optimal. However, there may exist a feasible LOT that does not belong to the subspace spanned by the columns of $P$. Hence, an optimal derived by this procedure may not be optimal globally in the sense of maximizing the energy compaction.

\section{INVESTIGATION RESULTS}

In this chapter simulation result is presented. In the first part, results of error concealment using MDC-DCT are presented. Although in this method by applying zero padding bit-rate is increased but high quality of received image causes to withdraw bit-rate increment. In the second part, the results of error concealment using MDCwavelet transform are shown. By using wavelet, there could be no blocking effect compared to DCT. In the third section, error concealment using MDC-LOT transform is presented. Since LOT is used there is no blocking effect and this method has high quality in low bit rates, which is useful in low channel capacity.

\subsection{Error concealment using MDC and DCT}

Zero padding is pre-processing method [22]. In this process, some zeros are added to the image until high quality reconstruction of the image is achieved although losing descriptions in the transmission channel. The zero padding process is presented in Fig. 4. At first DCT is applied to whole image. Then some numbers of zeros are added to rows and columns. Inverse DCT is later used to map the data to spatial domain. Hence, the correlation of image is increased in this method. If there are losses in the channel, by using received descriptions, lost descriptions can be constructed in high quality. This method increases the bit rate of transmission, but we can trade bit rate increment to the quality of reconstructed image.

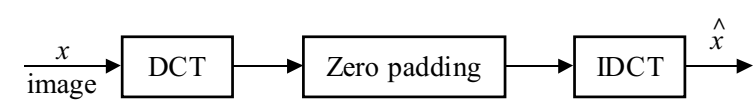

Fig. 4. Zero padding

In the simulation, Lena gray level $512 \times 512$ image is used. At first DCT is applied to the image then 108 lines zero are added to end of rows and columns. Inverse DCT is taken to obtain zero padded image in the spatial domain $\hat{X}$. At the next step as method presented in Fig. 5 four subimages are extracted from downsampled zero padded image. Downsampling method is shown in Fig. 6. From each subimage, DCT transform is performed and by using MDSQ with spread 3, eight descriptions are obtained from four subimages. These descriptions are then coded and sent through channels. At the receiver by taking average from received data, lost descriptions can be reconstructed. Therefore, error conceals from image. Then, the reverse process of above is conducted, two descriptions are added together then by using inverse DCT, the zero padded image is obtained after downsampling.

Table 1 shows average PSNR values of reconstructed images with different bit rate and lost descriptions. The values in the parentheses are standard deviation. Table 2 is average PSNR and standard deviation for [23]. In this paper, MDSQ with spread 3 is applied. By comparing the results of the proposed method with [23], minor decrement in PSNR is achieved even though losing some descriptions at the respective bit rate. Furthermore, standard deviation values in Table 1 are smaller than in Table 2. For example, if one description is received (seven descriptions are lost) in $0.58 \mathrm{bpp}$ (bit per pixel), the PSNR is $27.01 \mathrm{~dB}$ but in [23] with $0.625 \mathrm{bpp}$ for one received description the PSNR value is $22.88 \mathrm{~dB}$. 
Table 1. Average PSNR values and standard deviation for proposed method

\begin{tabular}{cccc}
\hline \multirow{2}{*}{$\begin{array}{c}\text { number } \\
\text { of lost }\end{array}$} & \multicolumn{3}{c}{ Bitrate } \\
\cline { 2 - 4 } descriptions & $2.31 \mathrm{bpp}$ & $1.15 \mathrm{bpp}$ & $0.58 \mathrm{bpp}$ \\
\hline 0 & 27.14 & 28.18 & 31.51 \\
1 & $31.43(0.05)$ & $28.12(0.07)$ & $27.13(0.1)$ \\
2 & $31.42(0.2)$ & $28.11(0.15)$ & $27.12(0.21)$ \\
3 & $31.32(0.18)$ & $28.09(0.17)$ & $27.11(0.2)$ \\
4 & $31.04(0.25)$ & $28.03(0.22)$ & $27.07(0.26)$ \\
5 & $31.11(0.2)$ & $28.00(0.17)$ & $27.05(0.03)$ \\
6 & $30.90(0.22)$ & $27.97(0.25)$ & $27.04(0.11)$ \\
7 & $30.67(0.07)$ & $27.90(0.05)$ & $27.01(0.08)$ \\
\hline
\end{tabular}

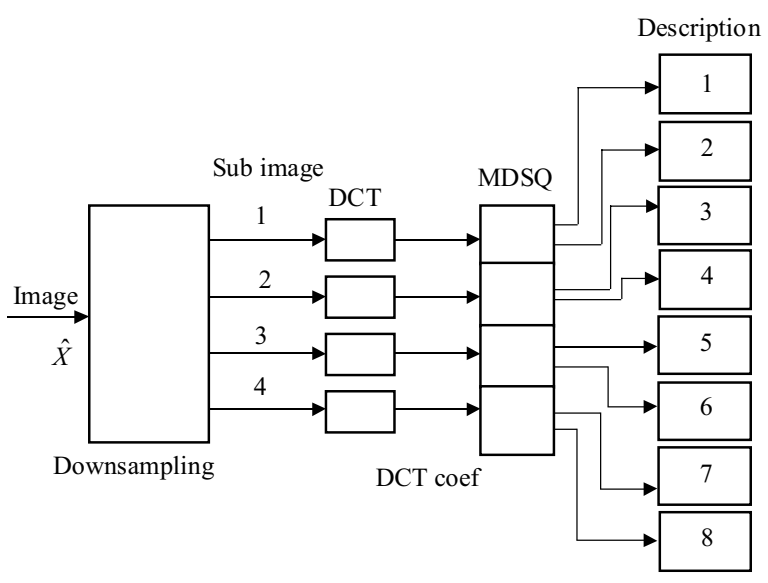

Fig. 5. Error concealment using MDC, zero padding and DCT

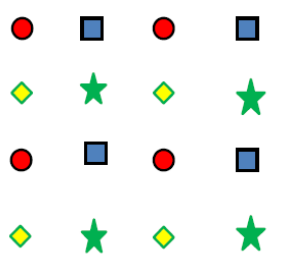

Fig. 6. Downsampling method

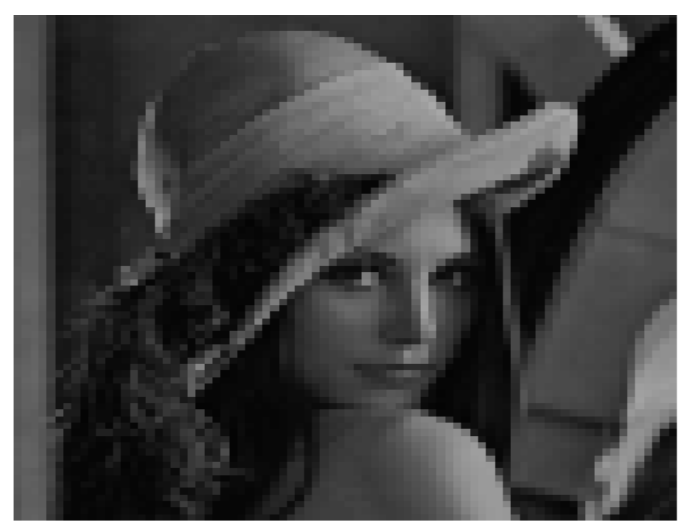

Fig. 7. Reconstructed image with 0ne received description in $0.58 \mathrm{bpp}$
Table 2. Average PSNR and standard deviation for [23]

\begin{tabular}{cccc}
\hline \multirow{2}{*}{$\begin{array}{c}\text { number } \\
\text { of lost } \\
\text { descriptions }\end{array}$} & $2 \mathrm{bpp}$ & $1 \mathrm{bpp}$ & $0.625 \mathrm{bpp}$ \\
\cline { 2 - 4 } & 29.80 & 27.67 & 26.44 \\
0 & $29.01(0.12)$ & $26.85(0.13)$ & $25.21(0.18)$ \\
1 & $28.99(0.71)$ & $26.36(0.58)$ & $24.57(0.86)$ \\
2 & $28.25(0.61)$ & $26.00(0.61)$ & $23.93(0.76)$ \\
3 & $27.96(0.95)$ & $25.71(0.68)$ & $23.78(1.1)$ \\
4 & $27.71(0.6)$ & $25.48(0.62)$ & $23.38(0.78)$ \\
5 & $27.50(0.8)$ & $25.40(0.96)$ & $23.24(1.39)$ \\
6 & $27.14(0.33)$ & $25.07(0.32)$ & $22.88(0.39)$ \\
7 & &
\end{tabular}

The higher compression used - in order to remove redundant data from image and transfer the information more efficiently - the lower is the (transmitted) bpp. But higher compression ratio (lower transmitted bpp) brings worsening of the reconstructed image, thus a higher compression means lowering the PSNR. Lena reconstructed image using the proposed method in $0.58 \mathrm{bpp}$ with 7 lost descriptions is shown in Fig. 7 while Fig. 8 shows Lena reconstructed image in [23] with 7 lost descriptions in 0.625 . For better understanding, the rate distortion plot of Table 1 and Table 2 is shown in Fig. 9. As shown MSE (mean square error) of the proposed method is lower than [23].

\subsection{Error concealment using MDC and Wavelet transform}

Orthogonal spline wavelet transform type with order two is applied to downsampled subimages and each of subimage is converted to two descriptions by using MDSQ. If some of the descriptions are lost, then by averaging the received descriptions, error can be concealed. By adding each two description together and using inverse wavelet, four subimages are obtained so upsampling process is used to reconstruct the whole image from subimages. The block diagram of the method is presented in Fig. 10.

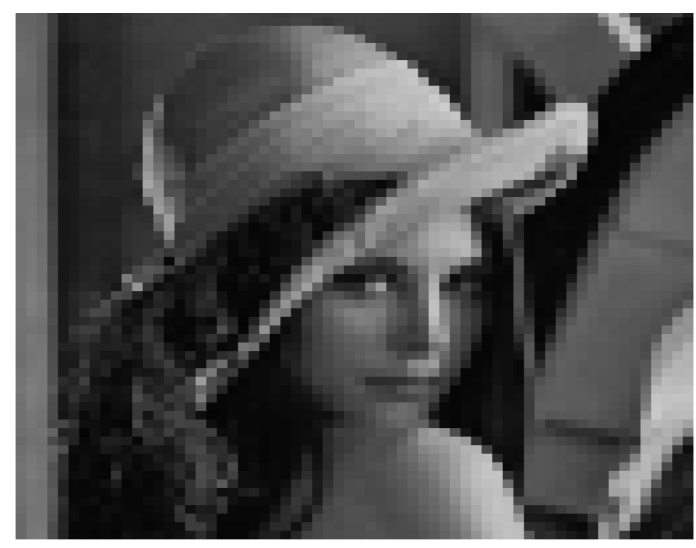

Fig. 8. Reconstructed image with one received description in 0.625 bpp [23] 


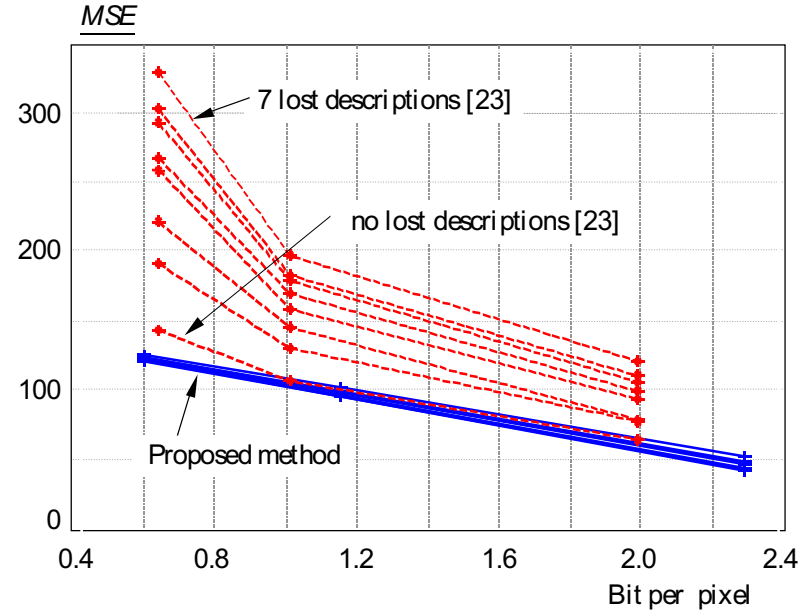

Fig. 9. Rate distortion performance for Table 1 proposed method (blue lines) and Table 2 (red lines) [23]. In each method from bottom to top number of lost descriptions are increasing.

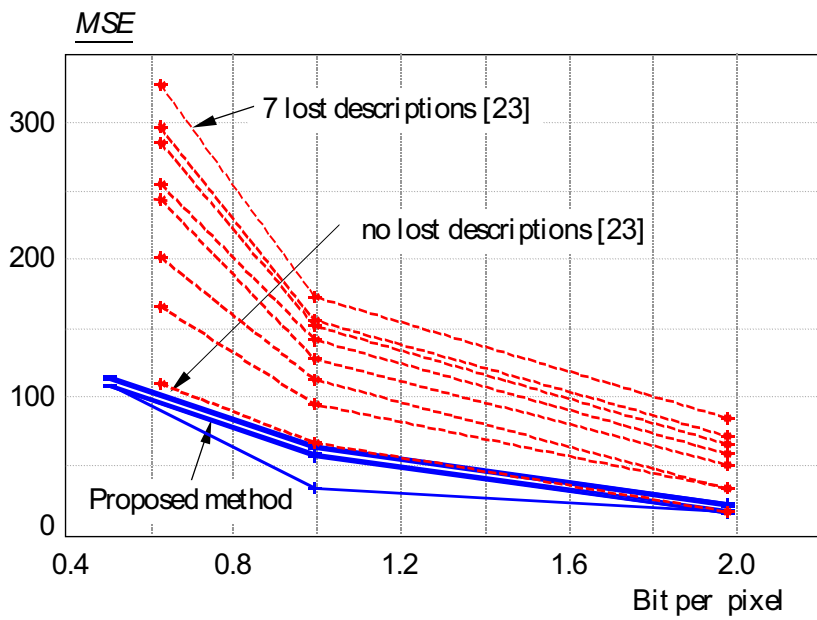

Fig. 11. Rate distortion performance for table 3 proposed method (blue lines) and Table 2 (red lines) [23]

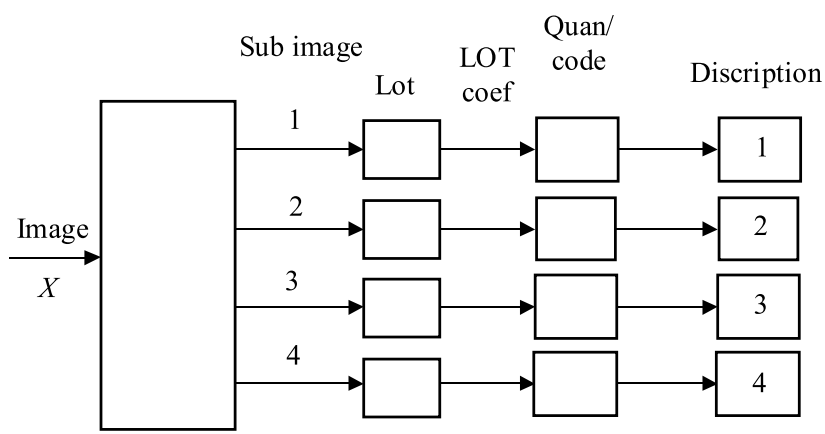

Downsampling

Fig. 13. Block diagram of error concealment using MDC and LOT

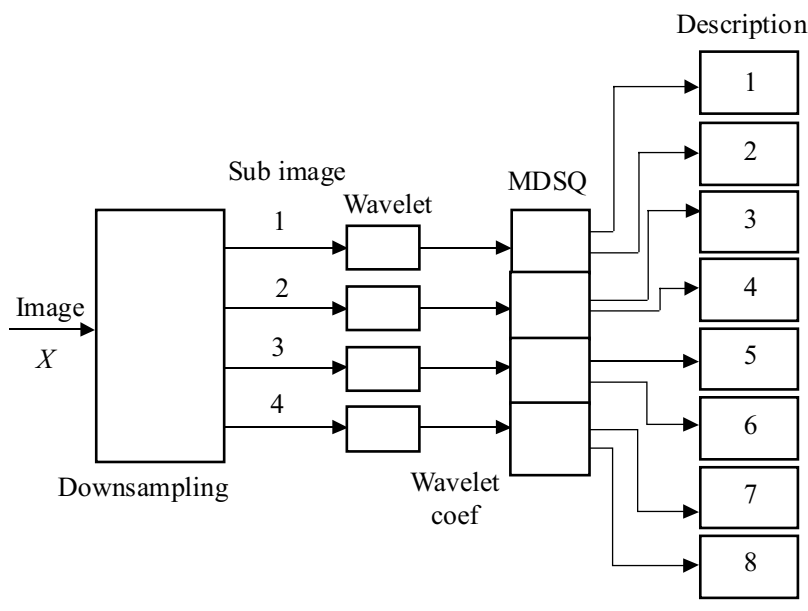

Fig. 10. Error concealment using MDC and wavelet

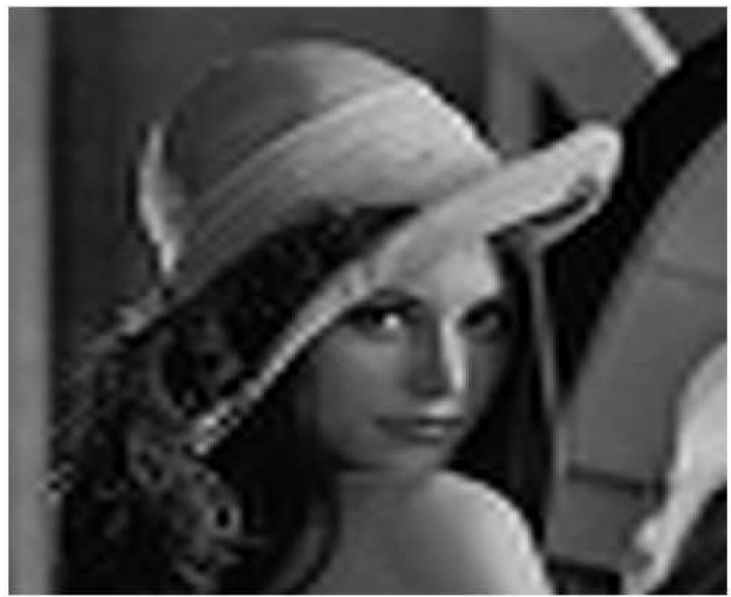

Fig. 12. Lena reconstructed image with one received description in 0.5 bpp using MDC and wavelet

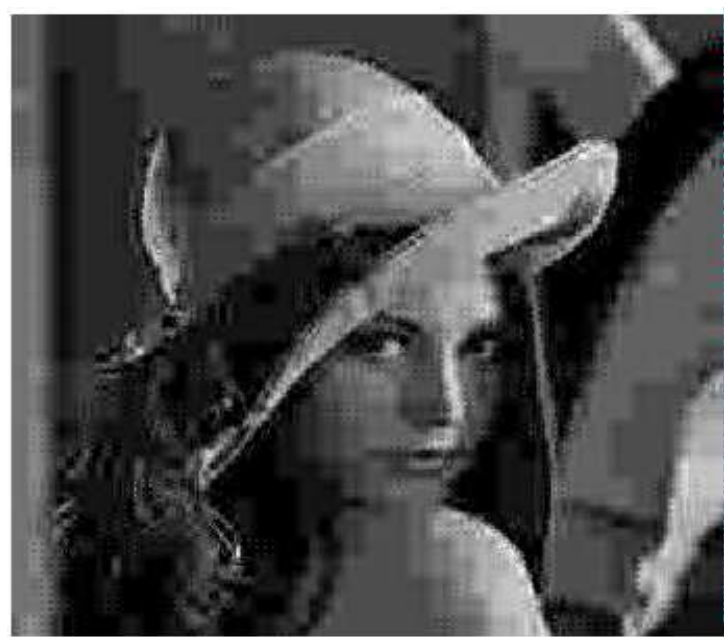

Fig. 14. Reconstructed image with three lost descriptions in 0.11 bpp with LOT method 


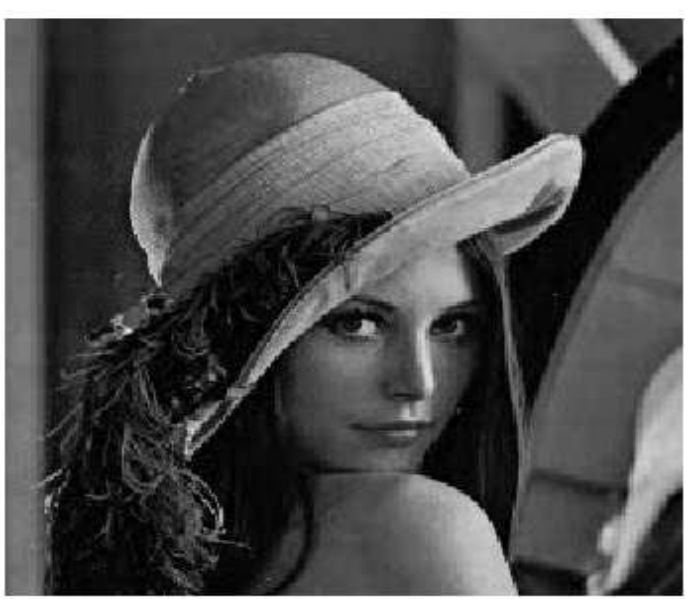

Fig. 15. Reconstructed image with three lost descriptions in 1 bpp with LOT method

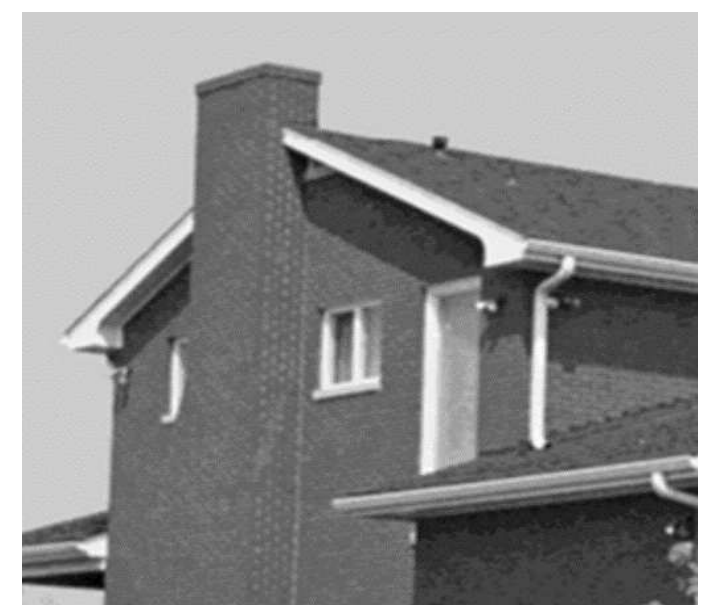

Fig. 17. Original image of house

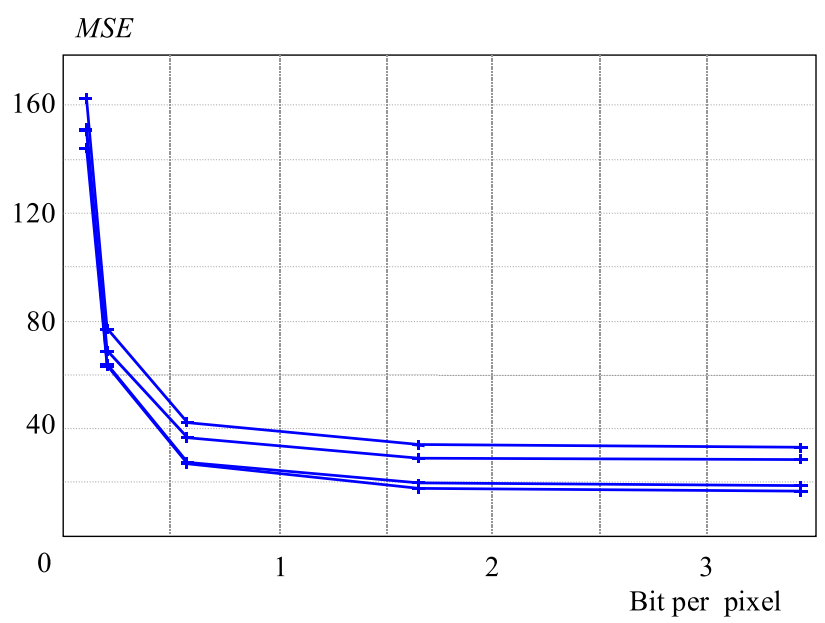

Fig. 19. Rate-distortion performance for error concealment using LOT and MDC for house image (Again in this method from bottom to top number of lost descriptions are increasing)

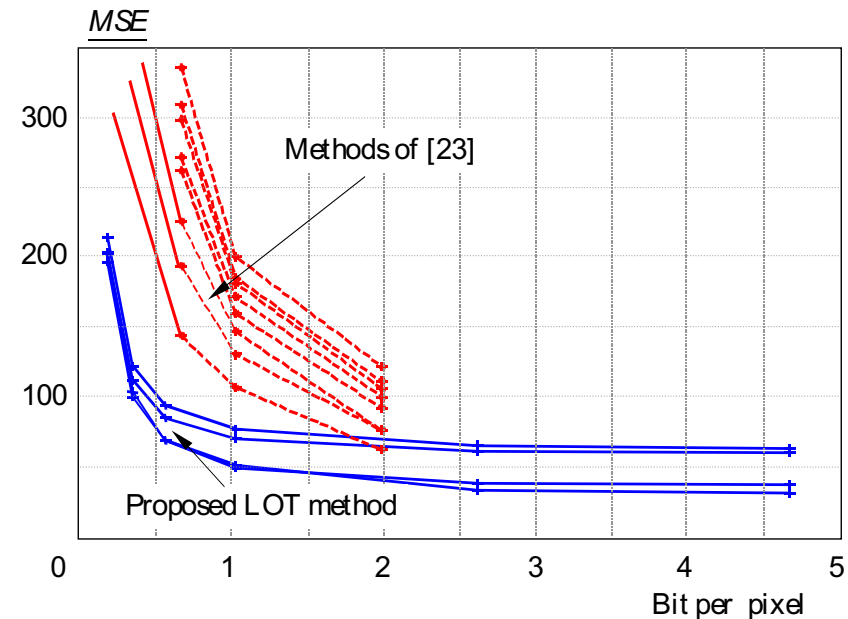

Fig. 16. Rate distortion performance for Table 4 proposed method (blue lines) and Table 2 (red lines) [23] (In each method from bottom to top number of lost descriptions are increasing)

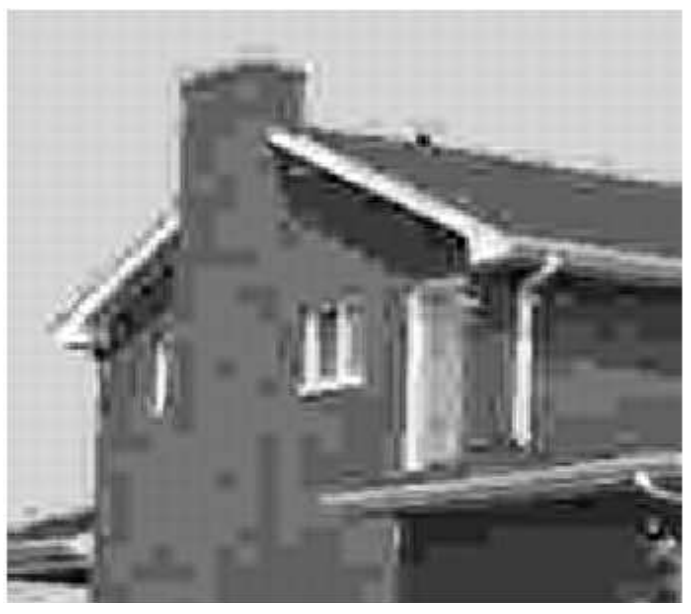

Fig. 18. Reconstructed image with one received description in 0.1 bpp with LOT method

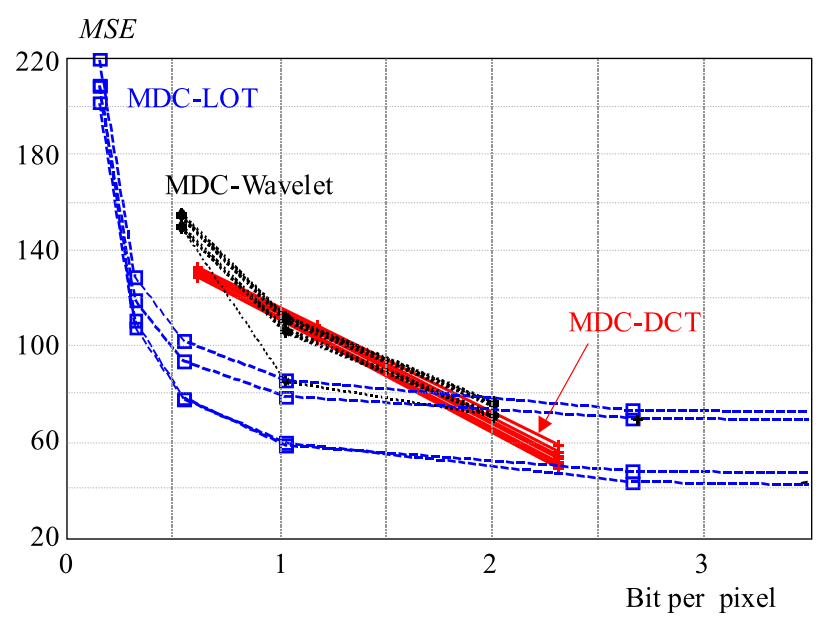

Fig. 20. Rate distortion performance of three proposed methods. In each method from bottom to top number of lost descriptions are increasing. 
178 A. Farzamnia - S. Kamilah - N. Fisal - S. A. Rahman: INVESTIGATION OF ERROR CONCEALMENT USING DIFFERENT ...

Table 4. Average PSNR and standard deviation for Lena image using MDC and LOT

\begin{tabular}{ccccccc}
\hline \multirow{2}{*}{$\begin{array}{c}\text { number } \\
\text { of lost }\end{array}$} & \multicolumn{5}{c}{ Bitrate } \\
\cline { 2 - 6 } descriptions & $0.11 \mathrm{bpp}$ & $0.28 \mathrm{bpp}$ & $0.51 \mathrm{bpp}$ & $1 \mathrm{bpp}$ & $2.66 \mathrm{bpp}$ & $4.8 \mathrm{bpp}$ \\
\hline 0 & 25.15 & 27.93 & 29.40 & 30.60 & 32.12 & 32.34 \\
1 & $25.01(0.2)$ & $27.81(0.3)$ & $29.38(0.4)$ & $30.50(0.31)$ & $31.63(0.51)$ & $31.80(0.39)$ \\
2 & $25.00(0.19)$ & $27.47(0.15)$ & $28.56(0.22)$ & $29.33(0.17)$ & $29.89(0.27)$ & $29.99(0.6)$ \\
3 & $24.78(0.1)$ & $27.14(0.2)$ & $27.17(0.34)$ & $28.95(0.29)$ & $29.64(0.41)$ & $29.77(0.32)$ \\
\hline
\end{tabular}

Table 3. Average PSNR and standard deviation for Lena image using MDC and wavelet

\begin{tabular}{cccc}
\hline \multirow{2}{*}{$\begin{array}{c}\text { number } \\
\text { of lost } \\
\text { descriptions }\end{array}$} & $2 \mathrm{bpp}$ & $1 \mathrm{bpp}$ & $0.5 \mathrm{bpp}$ \\
\cline { 2 - 4 } & 29.86 & 28.02 & 26.49 \\
0 & $29.85(0.03)$ & $28.01(0.05)$ & $26.48(0.1)$ \\
1 & $29.81(0.13)$ & $27.98(0.16)$ & $26.47(0.14)$ \\
2 & $29.77(0.21)$ & $27.96(0.24)$ & $26.45(0.18)$ \\
3 & $29.57(0.3)$ & $27.82(0.27)$ & $26.36(0.32)$ \\
4 & $29.53(0.25)$ & $27.80(0.23)$ & $26.34(0.19)$ \\
5 & $29.49(0.15)$ & $27.77(0.14)$ & $26.32(0.15)$ \\
6 & $29.47(0.07)$ & $27.75(0.09)$ & $26.30(0.06)$ \\
7
\end{tabular}

Table 5. Average PSNR for house image using MDC and LOT

\begin{tabular}{cccccc}
\hline \multirow{2}{*}{$\begin{array}{c}\text { number } \\
\text { of lost }\end{array}$} & \multicolumn{5}{c}{ Bitrate } \\
\cline { 2 - 6 } descriptions & 0.1 bpp & 0.2 bpp & 0.57 bpp & 1.65 bpp & $3.4 \mathrm{bpp}$ \\
\hline 0 & 26.50 & 30.1 & 33.82 & 35.58 & 35.89 \\
1 & 26.33 & 30.06 & 33.69 & 35.19 & 35.42 \\
2 & 26.30 & 29.70 & 32.46 & 33.42 & 33.55 \\
3 & 26.00 & 29.22 & 31.84 & 32.79 & 32.94 \\
\hline
\end{tabular}

Table 3 shows average PSNR and standard deviations for Lena image in different lost descriptions and bit rates. As you compare Table 3 and Table 2, it can be observed that PSNR values are higher than in Table 2 . The rated of PSNR decrement are lower than Table 2. For better understanding, rate-distortion plot is presented in Fig. 11. It is obvious that at every bit rate, MSE values of proposed method are much lower than [23] and sustainable even descriptions are lost.

In Fig. 12, the Lena image is reconstructed with only one received description ( 7 descriptions lost) in 0.5 bit per pixel. Comparing with Fig. 7 there is obviously no blocking effect, since the wavelet was used.

\subsection{Error concealment using MDC and LOT}

One of the benefits of LOT is that it has no blocking effect. Due to that, LOT is able to produce good image reconstruction quality even at low bit rate (around $0.1 \mathrm{bpp})$.
At high bit rate (somehow more than $2 \mathrm{bpp}$ ), the quality of reconstructed image is saturated and does not improve so much.

Nevertheless, in other transforms such as DCT and wavelet when bit rate increases (more than $2 \mathrm{bpp}$ ) the quality also increases.

It was found that in low capacity channel LOT method performs better than the others. Block diagram of this method is presented in Fig. 13. Again Lena image is downsampled to four subimages then lapped orthogonal transform applied to them and quantized and coded through the channel. At the receiver side inverse of this process utilized and if some of the descriptions have been lost by taking average of received descriptions lost data can be reconstructed. In Table 4 average PSNR and standard deviation for Lena image in LOT method are shown.

As it is obvious from $2.66 \mathrm{bpp}$ to 4.8 , the PSNR values do not change so much. Even so, in very low bit rate such as 0.11 bpp the PSNR value is high. In Fig. 14, reconstructed image at 0.11 bpp with 3 lost descriptions is shown. As illustrated there is no blocking effect and high PSNR values are achieved at low bit rates. In Fig. 15, reconstructed image in $1 \mathrm{bpp}$ with 3 lost descriptions is presented. In Fig. 16, rate-distortion plot of Table 2 and 4 is shown. At low bit rates MDC-LOT has the smallest MSE when compared to [23]. By increasing the bit rate from $2 \mathrm{bpp}$ to $4.8 \mathrm{bpp}$, the MSE does not change much as shown in Fig. 16.

This method is applied for house $512 \times 512$ pixel grey level image. In Fig. 17 the original image is shown. In Table 5 the average PSNR in different number of lost descriptions is presented. In Fig. 18, the house image is reconstructed with one received description in $0.1 \mathrm{bpp}$. Figure 19 shows the rate-distortion plot for house image.

In Fig. 20 rate-distortion of three methods with Lena image are illustrated in one plot. As it is obvious the in low bit rates MSE of MDC-LOT is lower than the others which means it has high performance in low capacity channels.

\section{CONCLUSION}

Combining MDC with LOT for source coding is an efficient way to eliminate redundant data and conceal errors accrued in channels. LOT is one of the transform coding methods that can map pixels of correlated image to uncorrelated symbols in transform domain. Unlike DCT, 
LOT has no blocking effects in the reconstructed images, and this characteristic can make the image more vivid. Due to use of MDC with LOT, MSE values of reconstructed image are very low in low bitrates but combining other transform codings such as DCT and wavelet with MDC can not have that much efficiency in low bit rates.

So this method is useful in low capacity channels. In addition, in proposed methods by losing descriptions PSNR values are decreased little by little and standard deviations of reconstructed descriptions are very small. Finally, from results it is obvious that not only proposed methods have high PSNR values but also have high subjective evaluation quality in low bit rates.

\section{REFERENCES}

[1] VAishampayan, V. A.: Design of Multiple Description Scalar Quantizers, IEEE Trans. on Information Theory 39 (May 1993), 821-834.

[2] VAishampayAn, V. A.-BATLLO, J. C. : Asymptotic analysis of multiple description quantizers, IEEE Trans. On Information Theory, vol. 44, pp. 278-284, Jan. 1998.

[3] SERVETTO, S. D.-RAMCHANDRAN, K.-VAISHAMPAYAN, V.-NAHRSTEDT, K.: Multiple description wavelet based image coding, in ICIP 98, 1998.

[4] WANG, Y.-ORCHARD, M.-REIBMAN, A. R.: Multiple Description Image Coding for Noisy Channels by Pairing Transform Coefficients, Proc. IEEE First Workshop on Multimedia Signal Processing, 1997.

[5] GOYAL, V. K.-KOVACEVIC, J.-AREAN, R.-VETTERLI, M. : Multiple Description Transform Coding of Images, in ICIP 98.

[6] ENRIQUe, J.-CANDAS, C.-AMARATUNGA, K. : Fast Estimation of Karhunen-Loeve Eigen Function using Wavelets, IEEE Trans. on Signal Processing (2001).

[7] JAIN, A.: A Fast Karhunen-loeve Transform for Digital Restoration of Images Degraded by White and Colored Noise, IEEE Trans. on Computers 26 No. 6 (June 1977).

[8] WATson, B.: Image Compression Using the Discrete Cosine Transform, NASA Ames Research Center.

[9] DAVIS, G.--NOSRATINIA, A. : Wavelet-Based Image Coding: An Overview, Applied and Computational Control, Signals and Circuits 1 No. 1 (Spring 1998).

[10] MALVAR, H. S.-STAELIN, D. H.: The LOT: Transform Coding without Blocking Effects, IEEE Trans. Acoust. Speech, and Signal Process. ASSP 37 (Apr 1989), 553-559.

[11] NORKIN, A.-BICI, M. O.-AKSAY, A.-BILEN, C.-GOTCHEV, A.-BOZDAGI AKAR, G.-EGIAZARIAN, K.-ASTOLA, J.: Multiple Description Coding and Its Relevance to 3DTV, in Three-Dimensional Television: Capture, Transmission, and Display (Haldun, M. Ozaktas and Levent Onural, eds.), Springer, Heidelberg.

[12] El GAMAL, A. A.-COVER, T. M.: Achievable Rates for Multiple Descriptions, IEEE Trans. on Inform. Theory IT-28 (Nov 1982).

[13] SHANNON, C. E. : A Mathematical Theory of Communication, Bell System Technical Journal 27 (July and Oct 1948), 379-423 and $623-656$

[14] OZAROW, L. H.: On a Source-Coding Problem with Two Channels and Three Receivers, Bell System Technical Journal 59 (1980), 1909-1921.

[15] GOYAL, V. K.-KOVACEviC, J.: Generalized Multiple Description Coding with Correlating Transforms, IEEE Trans. on Information Theory 47 No. 6 (Sep 2001), 2199-2224.
16] MALVAR, H. S.-STAELIN, D. H.: Reduction of Blocking Effects in Image Coding using Lapped Orthogonal Transform, ICASSP 88, Int. Conf. on Acoustic Speech and Signal Process., NY, Apr 1988, pp. 781-784.

17] MALVAR, H. S. : Signal Processing with Lapped Transforms, Artech House, Norwood, MA, 1992.

18] JAYANT, N. S.-NOLL, P.: Digital Coding of Waveforms, Prentice-Hall, Englewood Cliffs, NJ, 1984.

19] CAssereau, P. M.-STAELin, D. H.—de JAGER, G. : Encoding of Images Based on a Lapped Orthogonal Transform, IEEE Trans. on Communication 37 (Feb 1989), 189-193.

[20] MALVAR, H. S.: Optimal Pre- and Post-Filters in Noisy Sampled-Data Systems, PhD Dissertation, Dept. Elec. Eng. MIT, Aug 1986.

[21] MALVAR, H. S.: A Pre- and Post-Filtering Technique for the Reduction of Blocking Effects, Picture Coding Symp, Stockholm, Sweden, June 1987.

[22] SHIRANI, S.-GALlANT, M.-KOSSENTINI, F.: Multiple Description Image Coding using Pre- and Post Processing, IEEE International Conference on Information Technology: Coding and Computing, vol. 1, Apr 2001, pp. 35-39.

[23] ZHIMING, X.-ZHIPING, L.-ANAMITRA, M. : Multiple Description Image Coding with Hybrid Redundancy, IEEE Asia Pacific conference on Circuits and Systems, APCCAS 2006, Dec 2006, pp. 382-385.

Received 6 March 2011

Ali Farzamnia received his BEng in telecommunication engineering from Islamic Azad University of Orumiyeh 2005. He got MSc in telecommunication engineering from university of Tabriz in 2008. He is currently working toward his PhD at Universiti Teknologi Malaysia in Telamatic research group, interested in network coding and source coding.

Sharifah K. Syed-Yusof received BSc (cum laude) in electrical engineering from Geoge Washington University USA in 1988 and obtained her MEE and PhD in 1994 and 2006 respectively from Universiti Tecknologi Malaysia. She is currently working as an associate professor at the Department of Radio Communication, Faculty of Electrical Engineering Universiti Teknologi Malaysia, interested in OFDMA based system, software define radio and cognitive radio.

Norsheila Fisal received her BSc in electronic communication from the University of Salford, Manchester, UK in 1984. MSc degree in telecommunication technology, and PhD degree in data communication from the University of Aston, Birmingham, UK in 1986 and 1993, respectively. Currently, she is a Professor with the Faculty of Electrical Engineering, University Technology Malaysia and Director of Telematic Research Group (TRG) Affiliated in Member of Institute Electrical and Electronic Engineers (IEEE), Member of Malaysian National Confederation of Computers (MNCC), Member of Institute For Information Processing (IFIP) and Member of Board of Engineer Malaysia.

Syed A. R. Abu-Bakar received BSc in Electrical Engineering from Clarkson University, New York, USA in 1990 and obtained his MSc from Georgia Institute of Technology, Atlanta, USA. in 1991 and PhD from University of Bradford, Bradford, UK in 1997. Currently he is an associate professor at the Department of Microelectronics and Computer Engineering as well as the head of computer vision, video and image procession research both at the Faculty of Electrical Engineering Universiti Teknologi Malaysia. 